\title{
Activité protéasique persistant dans les laits stérilisés $\left.{ }^{*}\right)$
}

\author{
par \\ J. GED et C. ALAIS \\ Service de Biochimie Appliquée - Université de Nancy I \\ 54037 Nancy cedex
}

\section{INTRODUCTION}

La présence d'une protéase dans le lait a été signalée pour la première fois par Babcok et Russel [1] en 1897. L'origine de cet enzyme a été discutée, mais il est admis aujourd'hui qu'il existe une protéase d'origine mammaire, ne provenant pas de la prolifération microbienne. Les travaux de Warner et Polis [2] ont montré que la protéase du lait est adsorbée sur la caséine native, d'où il est difficile de la séparer ; elle se trouve dans le précipité formé à $\mathrm{pH} 4,6$ et elle se manifeste par une protéolyse qui provoque, d'une part, une diminution de la viscosité des solutions et d'autre part, la libération de peptides solubles à $\mathrm{pH}$ 4,6 et dans l'acide trichloracétique. Une purification plus poussée que la précédente a été effectuée par Dulley [3] ; il attribue à l'enzyme un poids moléculaire élevé, 100000. Cette protéase est retrouvée également associée aux micelles séparées du lait par ultra-centrifugation, d'où elle a été purifiée par Reimerdes et Klostermeyer $[4,5]$; la caséine $\beta$ serait le substrat préféré ; ce processus de protéolyse interviendrait dans la conversion de la caséine $\beta$ en caséine $\gamma$ et d'autres constituants mineurs de la caséine isoélectrique. L'enzyme séparé par ces auteurs aurait une double spécificité ; l'une concerne les liaisons peptidiques impliquant le groupe carboxylique de la lysine et de l'arginine, ce qui l'apparenterait aux endopeptidases du type trypsine ; l'autre concerne une activité de type leucine-aminopeptidase. Kaminogawa et al. $[6,7,8]$ pensent que la protéase naturelle du lait est identique à la plasmine, enzyme à sérine du groupe de la trypsine, active en milieu légèrement alcalin et de poids moléculaire 48000 ; ils ont en outre isolé du lait une seconde protéase de caractère acide ( $\mathrm{pH}$ optimum 3,5, poids

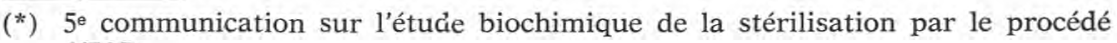
ATAD, 
moléculaire 36000 ), voisine de la cathepsine D. La protéase du lait devrait appartenir au groupe (EC.3.4.21.x.).

Cette protéase n'a pas été considérée, pendant longtemps, comme ayant de l'importance pratique ; on croyait que son activité était très faible au $\mathrm{pH}$ du lait ou du caillé de fromagerie et qu'en pratique elle ne jouait un rôle que dans la dégradation de la caséine en milieu alcalin, par exemple dans les colles. Son importance s'est beaucoup accrue avec le développement des procédés de stérilisation U.H.T. et l'apparition du phénomène de gélification du lait et de modification du goût après de longues durées de stockage à température ordinaire. Il a été écrit qu'il s'agit d'un phénomène purement physico-chimique $[9,10]$; cependant l'hypothèse biochimique est plus plausible; la gélification résulterait d'une activité protéolytique lente et limitée par la protéase du lait ou des protéases microbiennes, ayant résisté au traitement thermique rapide et s'étant réactivées $[11,12,13]$. Les résultats récents de Corradini [14] obtenus par électrophorèse et par la détermination des formes de l'azote est en faveur de cette hypothèse.

On relève des discordances en ce qui concerne le $\mathrm{pH}$ optimum d'activité de la protéase du lait, des valeurs s'échelonnant de 6 à 9 ont été avancées. Il en va de même pour l'effet des traitements thermiques. La présence d'un inhibiteur thermolabile peut expliquer l'accroissement de la protéolyse après une pasteurisation modérée du lait, comme l'ont montré Kiermeier et Semper [15]. Il n'y a pas à notre connaissance, de travaux démontrant la réactivation de cette protéase, après un traitement thermique.

Le développement de la pratique de la conservation du lait réfrigéré a attiré l'attention sur des protéases très thermorésistantes produites par les bactéries psychrotrophes du lait. Adams et al. $[16,17]$ ont trouvé ainsi des protéases résistantes $10 \mathrm{~s}$ à $149^{\circ} \mathrm{C}$ et ils pensent qu'elles pourraient, elles aussi, jouer un rôle dans les altérations du lait U.H.T., qu'il s'agisse de gélification ou de goût amer ; cependant, ces protéases peuvent être inactivées à température moyenne pendant une longue durée $\left(65^{\circ} \mathrm{C}, 60 \mathrm{mn}\right)$ par un processus qui ne doit pas être purement thermique.

L'activité protéolytique dans le lait cru de traite aseptique et dans le lait pasteurisé a été récemment étudiée par Noomen [18] par la mesure de l'azote soluble dans le surnageant résultant de la coagulation par la présure, l'incubation ayant lieu en anaérobiose et en présence d'un antiseptique. L'activité est très variable dans les laits individuels ; la pasteurisation accroît la protéolvse ; l'examen électrophorétique montre que la caséine $\beta$ est plus rapidement dégradée que la caséine $\alpha_{\mathrm{s}}$.

L'activité de la protéase du lait a été mise en évidence et mesurée de plusieurs manières : diminution de la viscosité [2], détermination 
de la tyrosine libérée selon Hull [19], titration par la méthode au formol jointe à l'électrophorèse en gel d'amidon selon Juffs [20].

En poursuivant nos recherches sur les effets biochimiques de la stérilisation ultra-rapide par friction, après avoir étudié la réactivation de la phosphatase alcaline [21], nous nous sommes intéressés à la persistance de l'activité protéasique après le chauffage dans diverses conditions. Pour cela, nous avons mis en pratique deux moyens d'investigation, d'une part la mesure de l'azote non protéique dans le lait et d'autre part, la mesure de l'azote non protéique dans les solutions de caséine extraite du lait stérilisé par précipitation acide. Dans la première méthode, les protéases microbiennes éventuellement présentes peuvent intervenir dans les résultats, si elles sont thermorésistantes ou réactivables. Dans la seconde, il est possible que l'on isole l'activité protéolytique due à l'enzyme natif qui est solidement fixé sur la caséine. En outre, nous avons effectué des examens électrophorétiques portant sur le lait et sur la caséine.

\section{MATERIEL ET METHODES}

Les conditions de stérilisation ultra-rapide par le procédé ATAD ont été précédemment indiquées [21]. La stérilisation a été effectuée aux températures de $130^{\circ} \mathrm{C}, 140^{\circ} \mathrm{C}, 150^{\circ} \mathrm{C}$ et $160^{\circ} \mathrm{C}$, les durées de chauffage étant respectivement de $0,3 \mathrm{~s}, 0,5 \mathrm{~s}, 0,8 \mathrm{~s}$ et $1,0 \mathrm{~s}$. Des essais de comparaison ont été effectués avec du lait U.H.T. industriel $\left(142^{\circ} \mathrm{C}, 2 \mathrm{~s}\right)$ et du lait stérilisé à $115^{\circ} \mathrm{C}$ pendant $20 \mathrm{mn}$.

La caséine a été précipitée selon les indications de Rowland [22] ; elle a été lavée deux fois à l'eau distillée sous agitation, puis remise en solution à $\mathrm{pH}$ 6,5 par addition de soude $0,1 \mathrm{~N}$, jusqu'au volume initial du lait. Cette solution doit être protégée contre le développement bactérien; nous avons essayé successivement des antibiotiques (pénicilline, tétracycline, terramycine) et des antiseptiques (thymol, formol, chloroforme). Finalement nous avons ajouté à la solution de caséine 1 p. 100 de chloroforme; il assure la stérilité durant l'expérience dans la plupart des essais et il ne réduit pas l'activité protéasique.

Pour les expériences avec le lait, nous avons utilisé l'antiseptique recommandé par Noomen [18] le thimerosal (merthiolate ou thiomersalate) à la dose de 0,01 p. 100 et utilisé le même dispositif d'incubation en anaérobiose.

L'incubation a eu lieu à $37^{\circ} \mathrm{C}$. A intervalles de temps réguliers on dose l'azote non protéique (NPN) dans le filtrat, après addition d'un volume de solution d'acide trichloracétique à $24 \mathrm{p} .100$ à un volume de solution de caséine ou de lait. L'azote est dosé par microKjeldahl. L'activité protéasique est exprimée par l'augmentation du NPN p. 100 de l'azote total. 
Des contrôles bactériologiques réguliers ont été effectués par culture sur plaques de gélose nutritive. La présence de 50 colonies $/ \mathrm{ml}$ justifiait l'élimination de l'essai.

Le $\mathrm{pH}$ a été contrôlé régulièrement ; il ne variait sensiblement pas. Les examens électrophorétiques ont été pratiqués en tubes de gel de polyacrylamide à 7,5 p. 100 , suivant la méthode de Maurer [23], adaptée à notre cas. Les échantillons de caséine lavée et lyophilisée sont dissous à la concentration de 1 p. 100 dans un tampon Tris $/ \mathrm{HCl}$ $\mathrm{pH} 8,0$; on dilue quatre fois avec l'urée $8 \mathrm{M}$ et le glycérol 60 p. 100 ; on ajoute une goutte de Mercapto-2-éthanol et on fait le dépôt sur le gel $(100 \mu \mathrm{l})$. La migration dure $2 \mathrm{~h}$ sous une intensité de 2,5 mA par tube. La coloration se fait avec le noir amido 1 p. 100 dans l'acide acétique 7 p. 100. La décoloration se fait avec de l'acide acétique 7 p. 100 .

\section{RESULTATS}

\section{Activité protéasique liée à la caséine}

La figure 1 présente les courbes de protéolyse des solutions de caséine. La première observation importante concerne la variabilité de l'activité liée à la caséine du lait cru (a). Dix expériences ont été réalisées avec dix laits de grand mélange différents ; dans sept d'entre elles les courbes obtenues sont assez groupées ; l'augmentation du NPN après $15 \mathrm{j}$ à $37^{\circ} \mathrm{C}$ se situe entre $1,8 \mathrm{p} .100$ et 3,5 p. 100. Dans une autre expérience (courbe 6) l'activité est nettement plus faible et dans deux autres expériences (non représentées sur la figure 1) la protéolyse a été plus rapide et beaucoup plus importante, avec une augmentation de NPN proche de $10 \mathrm{p} .100$ après $15 \mathrm{j}$; dans ce dernier cas, on peut suspecter l'intervention de protéases microbiennes.

Une autre observation concerne la forme des courbes. Elles montrent presque toutes un ralentissement de la protéolyse, après 3 ou $6 \mathrm{j}$ d'incubation, suivi d'une reprise plus rapide.

Après le traitement thermique par friction à $130^{\circ} \mathrm{C}, 140^{\circ} \mathrm{C}, 150^{\circ} \mathrm{C}$ et même $160^{\circ} \mathrm{C}$, l'activité protéolytique liée à la caséine persiste, mais elle est réduite. Les essais comparatifs permettent d'apprécier grossièrement la réduction; si l'on prend 100 pour la caséine du lait cru, on aura, pour la caséine du lait stérilisé : de 70 p. 100 à 80 p. 100 à $130^{\circ} \mathrm{C}$, de 60 p. 100 à 75 p. 100 à $140^{\circ} \mathrm{C}$, de 30 p. 100 à 40 p. 100 à $150^{\circ} \mathrm{C}$ et environ de 20 p. 100 à $160^{\circ}$ C. Dans le lait U.H.T. on observe une activité résiduelle plus faible que les précédentes : environ 15 p. 100 ; par contre, aucune activité n'a été observée avec la caséine du lait autoclavé.

Les courbes de protéolyse après stérilisation (b, c, d) conservent la même allure que celles de la caséine du lait cru. La caséine n'ayant donné au'une faible protéolyse avant stérilisation (a, 6) garde cette caractéristique après le traitement thermique $(d, 6)$. 


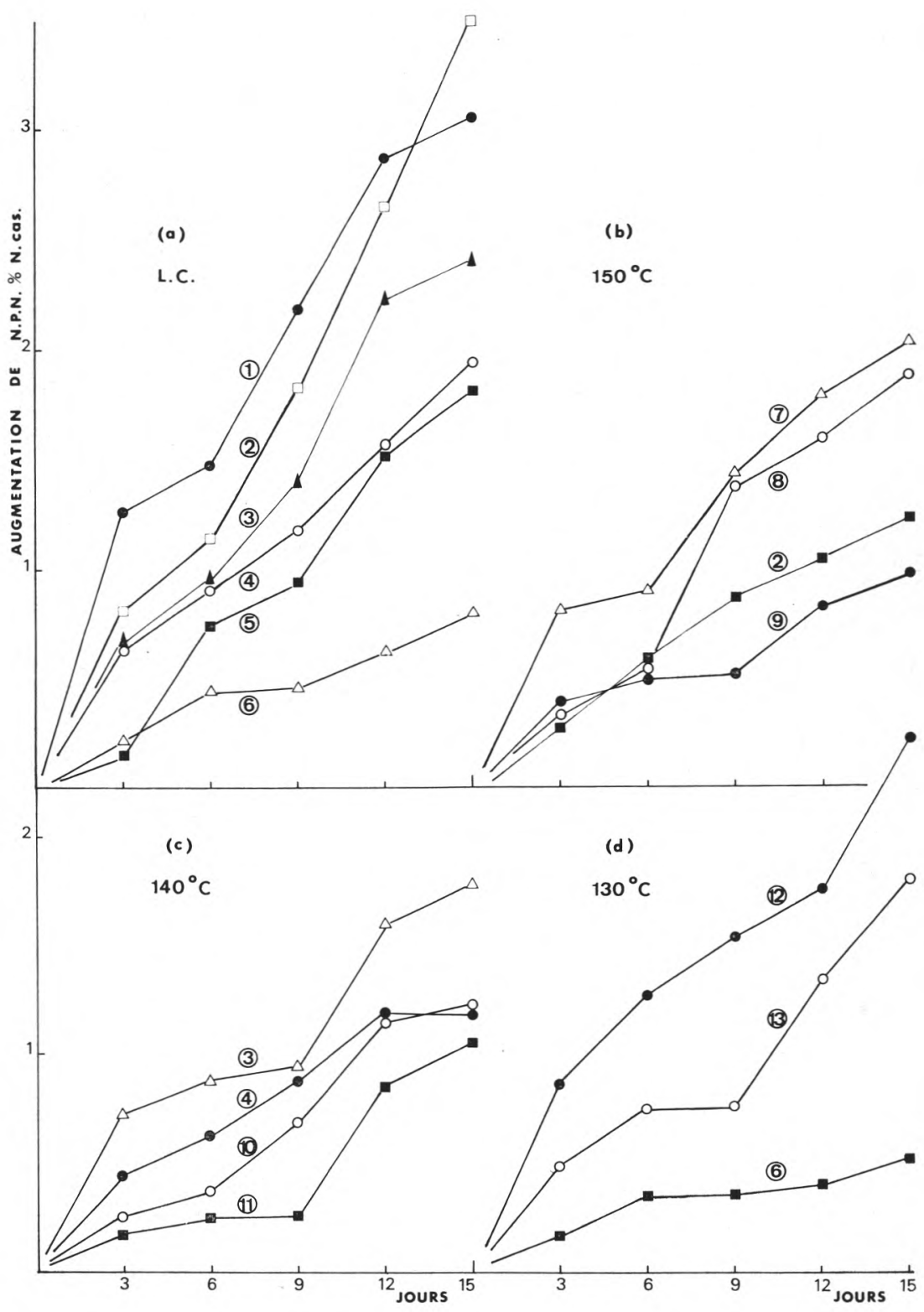

fig. 1

Activité protéolytique liée à la caséine cu lait cru (L.C.) et du lait stérilisé par friction à $130^{\circ} \mathrm{C}, 140^{\circ} \mathrm{C}$ et $150^{\circ} \mathrm{C}$; incubation à $37^{\circ} \mathrm{C}$. Les courbes portant le même numéro correspondent au même lait, cru ou stérilisé. 


\section{Etude électrophorétique de la dégradation de la caséine}

Les diagrammes d'électrophorèse en gel de polyacrylamide des caséines provenant de lait cru et de laits stérilisés montrent que lorsque la protéolyse se produit, c'est aux dépens de la caséine $\beta$ (fig. 2).

La dégradation de la caséine $\beta$ est prononcée dans le cas du lait cru et des laits stérilisés par friction à $130^{\circ} \mathrm{C}, 140^{\circ} \mathrm{C}$ et $150^{\circ} \mathrm{C}$;

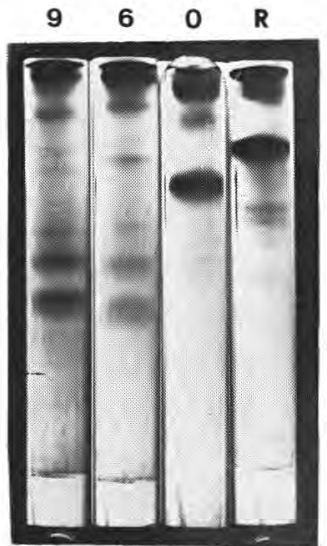

C ru

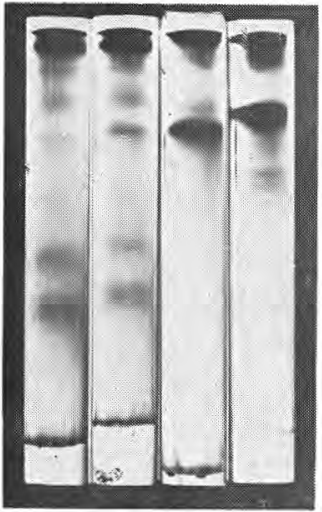

150

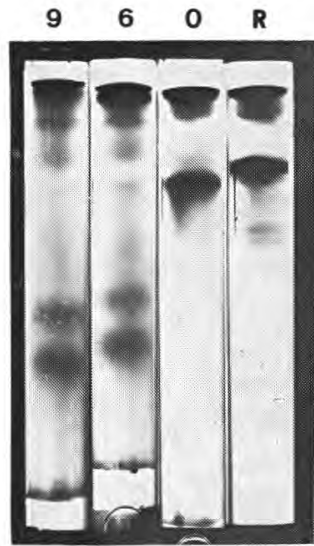

130

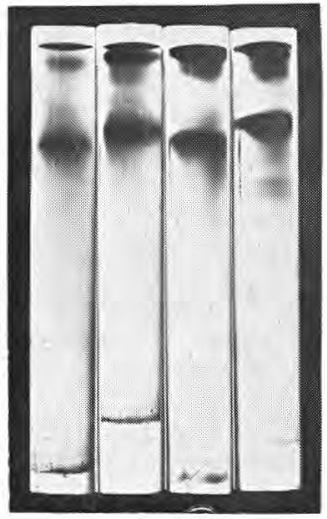

160

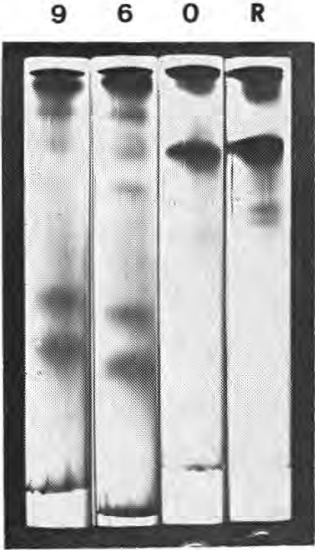

140

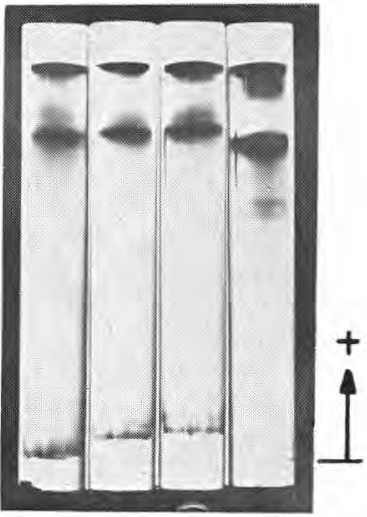

U H T

fig. 2

Diagramme d'électrophorèse en gel de polyacrylamide des c.séines du lait cru et des laits stérilisés. $\mathrm{R}$ : caséine de référence ; 0 : examen après la mise en solution ; 6 : examen après $6 \mathrm{j}$ d'incubation ; 9 : examen après $9 \mathrm{j}$. 
par contre elle n'est pas détectable à l'œil dans le cas du lait U.H.T., ni du lait stérilisé à $160^{\circ} \mathrm{C}$ ou à l'autoclave (l'image de ce dernier ne se trouve pas sur la figure 2).

Les diagrammes de la figure 2 montrent que le même type de dégradation se produit dans la caséine du lait cru comme dans la caséine du lait stérilisé par friction. La bande de la caséine $\beta$ disparaît et deux bandes plus lentes et bien colorées apparaissent ; dans le cas du lait cru il y a plus de bandes apparues au cours de la protéolyse que dans le cas des laits stérilisés. Danj les diagrammes d'électrophorèse des caséines des laits stérilisés, au temps $\mathrm{O}$ apparaît la bande de la $\beta$-lactoglobuline un peu au-dessus de celle de la caséine $\beta$; cette bande semble persister après l'incubation.

\section{Activité protéolytique dans le lait}

On a observé dans le lait cru et dans les laits stérilisés par friction une activité protéolytique au cours de l'incubation en anaérobiose à $37^{\circ} \mathrm{C}$ en présence d'antiseptique. Les courbes de libération du NPN de la figure 3 sont comparables à celles des caséines (fig. 1) ;

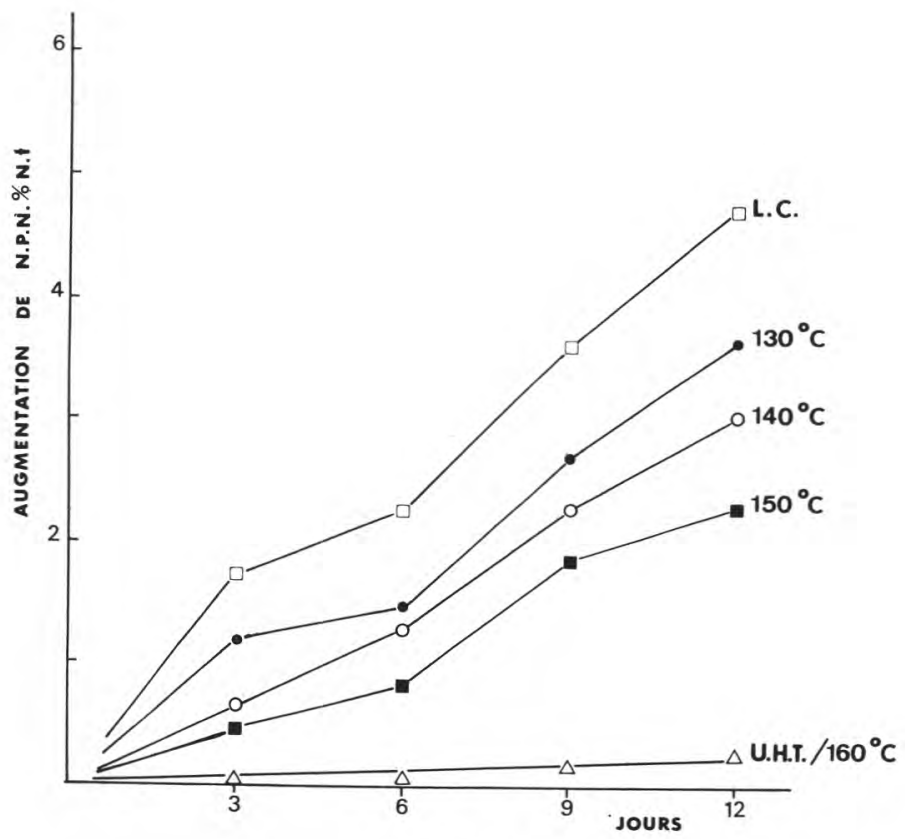

fig. 3

Activité protéolytique dans le lait conservé à $37^{\circ} \mathrm{C}$ en présence d'un antiseptique. LC : lait cru ; $130^{\circ} \mathrm{C}, 140^{\circ} \mathrm{C}$ et $150^{\circ} \mathrm{C}$ : même lait stérilisé par friction (il correspond à la première série du tableau 1 ). 
dans le cas du lait cru les valeurs de NPN p. $100 \mathrm{~N}$ total sont plus élevées et surtout elles sont beaucoup plus régulières ; dans quatre expériences on a relevé après 12 j 4,75 p. 100 ; 5,04 p. 100, 5,71 p. 100 et 5,82 p. 100 d'augmentation.

Après le traitement thermique à $130^{\circ} \mathrm{C}, 140^{\circ} \mathrm{C}$ et $150^{\circ} \mathrm{C}$ l'activité protéasique résiduelle est du même ordre de grandeur que dans le cas des caséines ; par contre à $160^{\circ} \mathrm{C}$, ainsi que dans le lait U.H.T., elle est plus faible (tab. 1).

TABLEAU 1

Activité protéolytique relative dans les laits stérilisés (*)

\begin{tabular}{l|c|c|c|c}
\hline & \multicolumn{2}{|c|}{ Première série } & \multicolumn{2}{c}{ Deuxième série } \\
\cline { 2 - 3 } & $6 \mathrm{j}$ & $12 \mathrm{j}$ & $6 \mathrm{j}$ & $12 \mathrm{j}$ \\
& & & & \\
& & & & \\
& & & & \\
Lait stérilisé par friction : & 87 & 86 & 63 & 73 \\
$130^{\circ} \mathrm{C}$ & 65 & 67 & 55 & 61 \\
$140^{\circ} \mathrm{C}$ & 30 & 38 & 36 & 47 \\
$150^{\circ} \mathrm{C}$ & - & - & 0 & 5 \\
$160^{\circ} \mathrm{C}$ & - & - & 2 & 5 \\
Lait U.H.T. & 0 & 0 & 0 & 0 \\
Lait autoclavé & & & & \\
\hline
\end{tabular}

(*) Incubation à $37^{\circ} \mathrm{C}$ en présence de thimerosal ; la valeur de l'augmentation du NPN dans le lait cru est prise égale à 100 .

La forme des courbes de protéolyse obtenues par incubation du lait ou de la caséine suggère la présence de plusieurs protéases actives. Pour obtenir une information complémentaire nous avons suivi la libération de NPN pendant des temps assez courts, dans une expérience résumée par la figure 4. Le lait cru (a) donne une courbe presque linéaire ; ce même lait additionné d'une faible dose de chymosine $(0,005$ unités $/ \mathrm{ml})$ donne une courbe (c) reproduisant la forme observée précédemment. Un essai témoin après stérilisation de ce lait à l'autoclave et addition de chymosine donne la courbe (b) avec palier caractéristique de la « réaction primaire » de cet enzyme sur la caséine. Il se trouve que la courbe (c) représente approximativement la somme des valeurs ayant donné les courbes (a) et (b). 


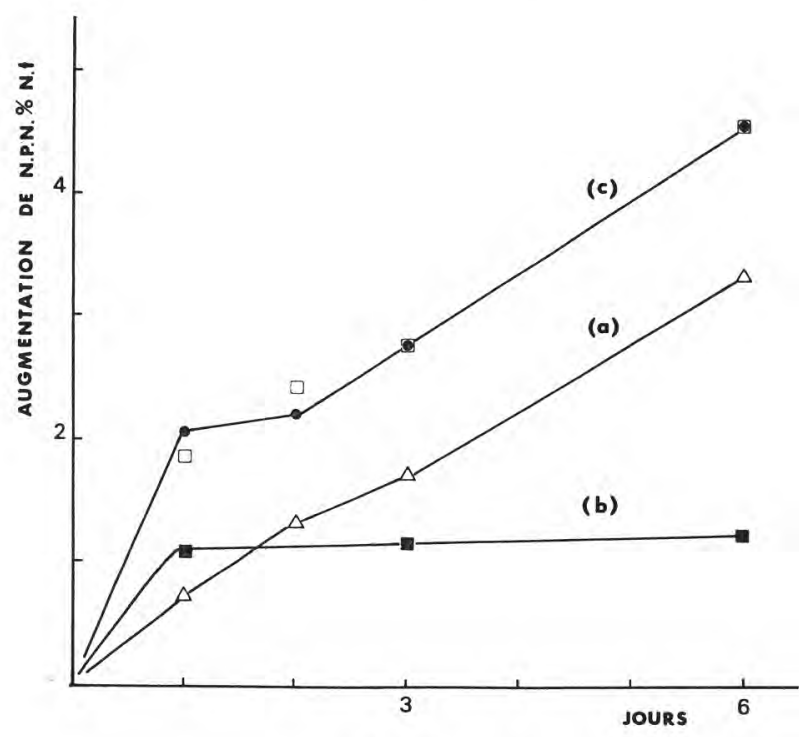

fig. 4

Activité protéolytique dans le lait conservé à $37^{\circ} \mathrm{C}$ en présence d'un antiseptique avec et sans addition de chymosine; (a) : lait cru ; (b) : lait autoclavé + chymosine ; (c) : lait cru + chymosine ; les carrés représentent la somme des valeurs prises sur les courbes (a) et (b).

\section{DISCUSSION}

La protéase ou le système protéolytique se trouvant dans le lait cru, présente une assez grande résistance au traitement thermique. L'enzyme de Warner et Polis [2] n'était inactivée qu'après un chauffage à $80^{\circ} \mathrm{C}$ durant $10 \mathrm{mn}$ de la solution de caséine, mais Kiermeier et Semper [15] ont indiqué que l'inactivation de la protéase du lait se produisait après un chauffage de $2 \mathrm{mn}$ à $70^{\circ} \mathrm{C}$.

Notre étude montre que l'activité protéasique n'est pas détruite lors de la stérilisation du lait à haute température pendant un temps très court. Comme pour la phosphatase alcaline [21] un traitement thermique de type U.H.T. dénature moins l'enzyme qu'un traitement de type pasteurisation, à plus basse température mais pendant un temps plus long.

L'activité protéasique que nous avons observée, après la stérilisation, est probablement plus une activité résiduelle que le résultat d'une réactivation, étant donné le niveau d'activité observé qui se situe beaucoup plus haut que celui que nous avions déterminé pour la phosphatase alcaline [21]. En outre, l'activité protéasique diminue 
lorsque la température du traitement par friction augmente. Le lait stérilisé par le procédé ATAD à $160^{\circ} \mathrm{C}$ se comporte à peu près comme le lait U.H.T. industriel. Dans le lait stérilisé à l'autoclave, aucune activité ne se manifeste.

Le développement de la protéolyse est plus lent dans les solutions de caséine que dans le lait ; mais dans tous les cas il s'agit d'une activité faible, révélant la présence de quantités très faibles de protéase ; il se peut cependant que les conditions physico-chimiques ne soient pas entièrement favorables ou encore que des inhibiteurs soient présents.

Le problème se pose de savoir à quelle protéase est due l'activité observée : la protéase naturelle seule ou des protéases produites par les bactéries se trouvant dans le lait cru ? Nous ne sommes pas partis de laits prélevés aseptiquement à la mamelle, mais de laits de grand mélange qui pouvaient renfermer une microflore psychrotrophe dont l'aptitude à produire des protéases thermorésistantes a été montrée par Adams et al. [16] ; cependant, on ignore si ces protéases peuvent se fixer fortement à la caséine, comme c'est le cas pour la protéase de Warner et Polis [2].

Les courbes de protéolyse obtenues avec la caséine précipitée à $\mathrm{pH} \mathrm{4,6} \mathrm{et} \mathrm{soigneusement} \mathrm{lavée} \mathrm{ont} \mathrm{une} \mathrm{forme} \mathrm{caractéristique} \mathrm{que}$ l'on retrouve après la stérilisation. Elles ne sont pas simples et il est possible que deux actions se superposent avec des vitesses différentes, mais il faut noter que le substrat préféré serait le même, la caséine $\beta$. Il est permis de penser que ces courbes traduisent essentiellement l'activité de la protéase naturelle qui pourrait exister sous deux formes, comme cela a été signalé dans l'introduction. Par contre, la protéolyse observée dans le lait incubé en présence d'antiseptique et qui libère une proportion plus grande de l'azote total sous forme de NPN pourrait être la somme des activités de la protéase naturelle et des protéases microbiennes non fixées sur la caséine.

Nous n'avons pas trouvé dans la littérature de rapports sur des travaux utilisant la caséine séparée du lait comme système enzymesubstrat ; mais nous y avons trouvé une récente communication de Noomen [18] sur la protéolyse dans le lait cru de traite aseptique et dans le lait pasteurisé ; cet auteur a observé de grandes variations individuelles, la libération de l'azote soluble après action de la chymosine allant du simple au double. Dans nos expériences sur le lait de mélange nous n'avons observé que peu de variation, mais dans notre cas il s'agit d'azote non protéique.

En ce qui concerne le niveau de l'activité protéolytique après le traitement thermique, on ne peut le relier simplement au taux d'enzyme non dénaturé. On sait, d'une part, que le chauffage modifie le substrat et favorise souvent la protéolyse et que, d'autre part, le lait contient des inhibiteurs de protéase thermolabiles, 
comme cela a été montré par Klostermeyer et al. [24, 25] ; mais les protéases à sérine (c'est probablement le cas de la protéase naturelle du lait) sont moins sensibles à ces effets dans le lait que les protéases acides ou les protéases à $\mathrm{SH}$.

Nos examens électrophorétiques montrant que la caséine $\beta$ est hydrolysée rapidement concordent avec ceux d'autres auteurs ; la protéase naturelle du lait n'hydrolyse pas la caséine $\alpha_{s}$ ou tout au moins elle ne l'hydrolyse que lentement $[18,26]$. Il a été avancé que la protéase associée aux micelles est probablement la cause de la formation des caséines $\gamma_{1}, \gamma_{2}$ et $\gamma_{3}$ par une hydrolyse limitée de la caséine $\beta[4,27]$. Il est possible que les bandes les plus lentes apparues au cours de l'hydrolyse (fig. 2) représentent ces constituants.

La gélification au cours d'une longue conservation du lait stérilisé par le procédé U.H.T. a été attribuée à une protéolyse limitée $[11,12,13,14]$. Il est possible que les mêmes accidents soient observés dans le cas du lait stérilisé par friction, bien que la protéolyse puisse se dérouler de façon différente. Des travaux en cours permettront peut-être d'éclaircir ce point.

\section{Ré s u m é}

Une activité protéasique mesurée par la libération d'azote non protéique persiste après la stérilisation ultra-rapide par friction (procédé ATAD) ; elle a été observée en présence d'un antiseptique à la fois dans le lait et dans la solution de caséine. L'étude électrophorétique montre que c'est la caséine $\beta$ qui est hydrolysée, avec formation de bandes de type $\gamma$. L'activité résiduelle décroît lorsque la température de stérilisation augmente. Dans le lait stérilisé à l'autoclave, aucune activité n'est décelable.

\section{S u m m a r y}

\section{PROTEASE ACTIVITY IN THE STERILIZED MILKS}

A proteolytic activity mesured by the liberation of non protein nitrogen (N.P.N.) persists after a ultra-rapid sterilization by friction (ATAD process). This activity was observed in the presence of an antiseptic in the milk and in the casein solution. The electrophoretic study show that $\beta$-casein is the substrate; it is hydrolized with the formation of bands similar to those of $\gamma$-casein. The residual activity decreases when the sterilization temperature increases. Not any proteolytic activity was disclosed in conventionnaly sterilized milk $\left(115^{\circ} \mathrm{C}, 20 \mathrm{mn}\right)$. 


\section{Remerciements}

Ces recherches ont été effectuées avec l'aide de la D.G.R.S.T. (contrats $n^{o s}$ 73.7.1398 et 75.7.0383). Nous remercions la Société Seffac pour la mise à disposition du stérilisateur ATAD.

Reçu pour publication en août 1976.

\section{Références}

[1] Babcock (S. M.) and Russel (H. L.) (1897). - Wis. Agr. Exp. Sta. 14th Ann. Rep., 161.

[2] Warner (R. C.) and Polis (E.) (1945). - J. Amer. Chem. Soc., 67, 529.

[3] Dulley (J. R.) (1972). - J. Dairy Res., 39, 1.

[4] Reimerdes (E. H.) und Klostermeyer (H.) (1974). - Milchwissenschaft, 29, 517.

[5] Reimerdes (E. H.), Mrowetz (G.) und Klostermeyer (H.) (1975). - Milchwissenschaft, 30, 271.

[6] Kaminogawa (S.), Sato (F.) and Yamauchi (K.) (1971). - Agr. Biol. Chem., $35,1465$.

[7] Kaminogawa (S.), Mizobuchi (H.) and Yamauchi (K.) (1972). - Agr. Biol. Chem., 36, 2163.

[8] Kaminogawa (S.) and Yamatchi (K.) (1972). - Agr. Biol. Chem., 36, 2351.

[9] Samel (R.), Weaver (R. W. V.) and Gammack (D. B.) (1971). - J. Dairy Res., $38,323$.

[10] Nakai (S.), Wilson (H. K.) and Herried (E. O.) (1964). - J. Dairy Sci., 47, 754.

[11] BJoRcK (L.) (1973). - Milchwissenschaft, 28, 291.

[12] Bengtsson (K.), Gardhage (L.) und IsaKsson (B.) (1973). - Milchwissenschaft, 28, 495.

[13] Fox (K. K.), Holsinger (C. H.), Posati (L. P.) and Pallansch (M. J.) (1967). J. Dairy Sci., 50, 952.

[14] CoRRadini (C.) (1975). - Milchwissenschaft, 30, 413.

[15] Kiermeier (F.) and Semper (G.) (1960). - Z. Lebensm. Untersuch. Forsch., 111,373 and 483.

[16] Adams (D. M.), Barrach (J. T.) and Speck (M. L.) (1975). - J. Dairy Sci., $58,828$.

[17] Barach (J. T.), Adams (D. M.) and Speck (M. L.) (1976), - J. Dairy Sci., $59,391$.

[18] Noomen (A.) (1975). - Neth. Milk Dairy J., 29, 153.

[19] Hull (M. E.) (1947). - J. Dairy Sci., 30, 881.

[20] Juffs (H. S.) (1975). - J. Dairy Res., 42, 277.

[21] Ged (J.), Alais (C.) (1976). - Lait, 56, 407.

[22] Rowland (S. J.) (1938). - J. Dairy Res., 9-42.

[23] Maurer (H. R.) (1971). - Disc-electrophoresis (de Gruyter, Berlin).

[24] Klostermeyer (H.), Rabbel (K.) und Reimerdes (E. H.) (1975). - Milchwissenschaft, 30, 194.

[25] Reimerdes (E. H.), Klostemeyer (H.) und SAYK (E.) (1976). - Milchwissenschaft, 31, 329.

[26] Yamauchi (K.) and Kaminogawa (S.) (1972). - Agr. Biol. Chem., 36, 249.

[27] Kaminogawa (S.) and Yamauchi (K.) (1974). - Agr. Biol. Chem., 38, 2343. 